\title{
Lu-177-Based Peptide Receptor Radionuclide Therapy for Advanced Neuroendocrine Tumors
}

\author{
Keunyoung Kim ${ }^{1} \cdot$ Seong-Jang $\mathrm{Kim}^{2}$
}

Received: 6 September 2017 / Revised: 31 October 2017 / Accepted: 1 November 2017 / Published online: 20 November 2017

(C) Korean Society of Nuclear Medicine 2017

\begin{abstract}
Peptide receptor radionuclide therapy (PRRT) is a systemic cytotoxic radiation therapy using a compound of $\beta$ emitting radionuclide chelated to a peptide for the treatment of tumor with overexpressed specific cell receptor such as somatostatin receptor subtype 2 (SSTR2) of neuroendocrine tumor (NET). Surgical resection should be performed for the curative treatment for NETs when it is feasible; however, a multi-disciplinary approach is needed when locally advanced or metastasized disease. PRRT with lutetium-177 (Lu-177)labeled somatostatin analogues, as a new treatment modality targeting metastatic or inoperable NETs expressing the SSTR2, have been developed and successfully used for the past two decades. As Lu-177 emits both $\beta$ - and $\gamma$-radiation, it has the ability as a theragnostic agent for NETs compared with only $\beta$-emitting yttrium-90 labeled PRRT. Several recent studies reported that $\mathrm{Lu}-177$ gave an overall positive response and improved the patients' quality of life. To fully exploit its potential, large comparative studies are needed for the assessment of distinct efficacies of Lu-177 labeled PRRT. Additionally, for extending the indications and developing new regimens of Lu-177-based PRRT, more dedicated clinical research is required.
\end{abstract}

Keywords Peptide receptor radionuclide therapy · Neuroendocrine tumors $\cdot$ Radiolabeled somatostatin analogues $\cdot$ Lu-177

Seong-Jang Kim

growthkim@daum.net

1 Department of Nuclear Medicine and Biomedical Research Institute, Pusan National University Hospital, Busan, South Korea

2 Department of Nuclear Medicine and Research Institute for Convergence of Biomedical Science and Technology, Pusan National University Yangsan Hospital, Yangsan, South Korea

\section{Introduction}

According to the United States Surveillance Epidemiology and End Results database and several other European databases from 1973 to 2007, they estimated the incidence of gastroenteropancreatic neuroendocrine tumors (NETs) at between 2.5 and 6.2 cases/100,000 population [1]. Over the past three decades, the incidence and prevalence of all NETs have shown a fivefold increased, greater than many other malignancies [2]. Surgical resection is the curative treatment for NETs; however, surgery is not feasible when NET is locally advanced or metastasized.

Overexpressed somatostatin receptor subtype 2 (SSTR2) on the cell membrane of tumor cell of NETs could be a key target molecule for peptide receptor radionuclide therapy (PRRT) with radiolabeled somatostatin [3]. Since the initial attempt of peptide receptor radionuclide therapy (PRRT) using In-111 octreotide in the 1990s, modified somatostatin analogue with a higher affinity for the SSTR2 and a more stable binding of radionuclide were developed. Lastly, labeled with the $\beta$ - and $\gamma$-emitting radionuclide lutetium-177 (Lu177) was shown to be very successful in achieving tumor reduction and survival in a rat model and several clinical trials. This review will address the possible advantages and clinical application of the use of Lu-177-labeled PRRT as a novel approach to radionuclide cancer therapy for advanced NETs.

\section{Neuroendocrine Tumors}

The NETs are a diverse group of malignancies deriving from the diffuse neuroendocrine cell system, with distribution almost everywhere in the human body. The gastrointestinal structures (72\%) and broncho-pulmonary systems (25\%) are the most frequent sites of NETs; less than $5 \%$ arise at other 
sites (e.g., thymus, breast and genitourinary system) $[4,5]$. NETs show heterogeneous clinical presentation and growth pattern and can be either functioning or nonfunctioning in nature. Some NETs are functioning tumors, which means that they produce and release excessive peptide or amines resulting distinct clinical symptoms, such as flushing, diarrhea, hypoglycemia, gastric ulcers or skin rash [6,7]. Although NETs arise from neuroendocrine cells, the majority of NETs are nonfunctioning tumors and are not associated with a distinct symptom of hormonal excess that may disturb their early detection. As a result, most patients present late with nonspecific symptoms, such as weight loss, bleeding or abdominal pain related to increased tumor mass and/or metastases [8]. According to these characteristics of NETs, $40 \%$ of the patients with NETs present with localized disease only, $20 \%$ of which are diagnosed with distant disease at the time of presentation [2]. Surgical removal is the only potentially curative therapy; however, many patients are diagnosed with inoperable state of NETs as mentioned above [2]. In locally advanced or metastatic NETs, a multi-disciplinary approach of cytoreductive strategies including octreotide therapy, chemotherapy, interferon alpha, interventional treatments or molecular targeted agents are needed [4]. PRRT may have benefits as a neoadjuvant or adjuvant therapy for unresectable or metastatic NETs [4].

\section{Somatostatin Receptor and Peptide Receptor Radionuclide Therapy (PRRT)}

On the secretary NETs cellular surface, five subtypes of SSTR are expressed which bind somatostatin. Among those five subtypes of SSTR, SSTR2 and SSTR5 mediate antisecretory effects of somatostatin and somatostatin analogues by inhibiting hormonal secretions in functioning NETs [9], and somatostatin binds with higher affinity to the SSTR2 [3]. Somatostatin could acts like an endogenous inhibitor of various hormones, such as serotonin, insulin, glucagon and gastrin [3]. Though NETs are usually slow growing, when these tumors have metastasized, treatment with somatostatin analogues results in reduced hormonal overproduction and symptomatic relief in most cases [3, 10]. For the objective tumor regression, however, treatment with somatostatin analogues is less successful $[4,11]$.

PRRT is a molecular-targeted radiation therapy binding the overexpressed receptors on tumors with high affinity and specificity. Peptides are usually classified as containing $<50$ amino acids, with a molecular weight (MW) of $\sim 5,500 \mathrm{Da}$; a peptide with 100 amino acids is considered more to be a protein [12]. A novel peptide analogue, Tyr3-octreotide (Fig. 1a), with a similar affinity profile for SSTRs was developed. Linked to a macrocyclic chelator, 1,4,7,10-tetraazacyclododecane-1,4,7,10-tetraacetic acid (DOTA), [DOTA0,Tyr3]-octreotide (DOTA-TOC) allows simple and stable radiolabeling $[13,14]$. Octreotate (Tyr3,Thr8octreotide), a newer analogue, which is identical to octreotide except that the C-terminal threonine amino alcohol is replaced by threonine with improved affinity for SSTR2, has been synthesized (Fig. 1b) [4]. The chelate analogue [DOTA0,Tyr3]octreotate (DOTA-TATE), which has a nine-fold higher affinity for SSTR2 than DOTA-TOC, can be labeled with radioisotopes [15]. Figure 1 depicts the conjugates which are based on octapeptides such as octreotide, octreotate, DOTA-TATE and Lu177-labeled DOTA-TATE. Since the first PRRT using yttrium90 (Y-90) DOTA-TOC was initially used in 1996 in a patient in Basel, Switzerland, PRRT has been developed and established as an effective therapeutic approach in the treatment of inoperable or metastatic NETs for almost two decades [10]. Since the year 2000, DOTA-TATE can be labeled with the $\beta$ - and $\gamma$-emitting isotope Lu-177 and has been used in clinical studies [4].

\section{Lutetium-177}

The general characteristics of Lu-177 are described in Table 1 . Lu-177-labeled somatostatin analogues have six- to sevenfold increased in affinity for the SSTR2 in a comparison of Y-90 loaded counterparts [16]. Besides, Lu-177 emits both $\beta$ - and $\gamma$-radiation, and this means that Lu- 177 acts as a theranostic isotope. As a $\beta$-emitting radionuclide, $\mathrm{Lu}-177$ is chelated to a peptide for the purpose of delivering cytotoxic radiation to a tumor with a maximal tissue penetration of $2 \mathrm{~mm}$. Lu-177labeled PRRT is more favorable because it emits lower energy $\left(\mathrm{E}_{\beta-\max } 0.49\right.$ vs $\left.2.27 \mathrm{MeV}\right)$ and has shorter emission range $(1.6 \mathrm{~mm}$ vs $11 \mathrm{~mm})$ of $\beta$-radiation compared with Y-90labeled PRRT [16, 17]. Therefore, Lu-177-labeled PRRT causes less damage to neighboring healthy cells in comparison to Y-90 [18]. Lu-177 also emits imagable $\gamma$-photons, which allows both imaging for monitoring and dosimetry of the same compound, whereas Y-90 emits only $\beta$-particles [16]. In terms of toxicity, Lu-177-labeled PRRT has proved to be safer than Y-90-based PRRT, due to the lower renal and bone marrow absorbed doses delivered with the commonly administered activities in the majority of cases [17].

\section{Process of Treatment}

Patient preparation includes an amino acid protection protocol for renal protection according to the guidance from the joint IAEA, EANM, and SNMMI recommendations as follows [4]. The protocol is composed of a co-infusion renoprotective amino acid mixture of positively charged amino acids, such as 5\% lysine $\mathrm{HCl}$ and $10 \% \mathrm{~L}$-arginine $\mathrm{HCl}$, which competitively inhibit the proximal tubular reabsorption of the radiopeptide and leads significantly to reduction in the renal absorbed dose $[19,20]$. 
Fig. 1 Structures and chelators of PRRT for radiolabeling based on octapeptides such as octreotide (a), octreotate (b), DOTA-TOC (c), DOTA-TATE (d), and Lu177-DOTA-TATE (e). According to the structure of Lu-177-labeled DOTA-TATE, DOTA is a chelator for the coupling of the Lu-177 and the somatostatin analogue (e) a $\mathrm{O}$

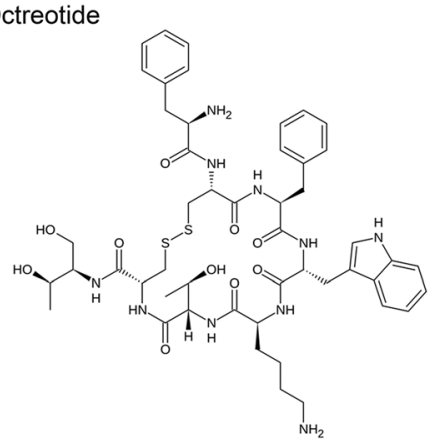

C DOTA-TOC

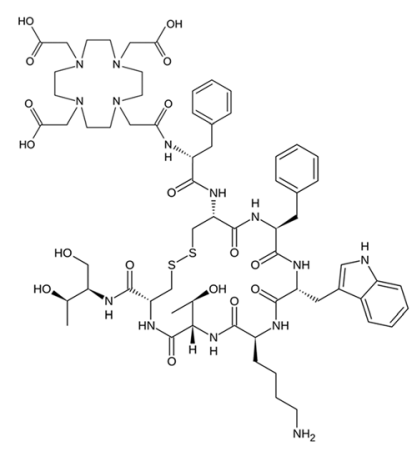

e Lu-177-DOTA-TATE

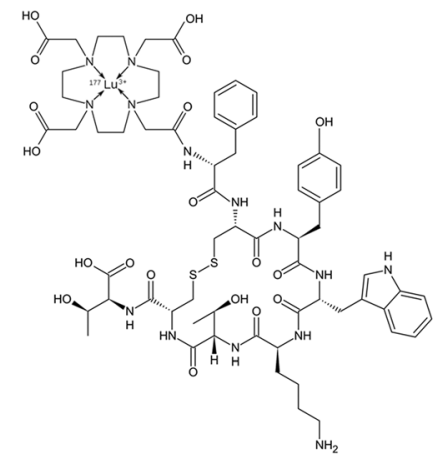

b Octreotate

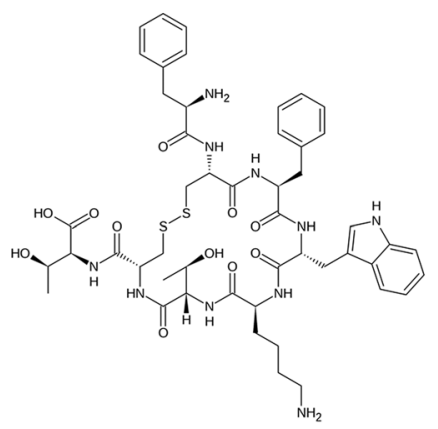

d Dota-tate

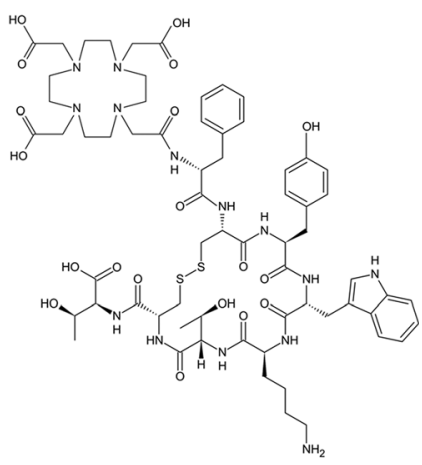

Several amino acid protective schemes composed of single-day or 3-day and 25 -g or 50-g protocols were recommended [4]. Regardless of the type of the protocol, the amino acid protocol should be started 30-60 min before administration of the radiopeptide and should be continued for $4 \mathrm{~h}$. Additive Gelofusine is recommended for further reduction of absorbed renal radiation dose [21, 22]; however, there have been concerns about allergic and anaphylactoid reactions [23,
24]. Vital parameters should be monitored during Gelofusine infusion [21].

Somatostatin analogue withdrawal for 4-6 weeks for longacting formulations and for at least $24 \mathrm{~h}$ for short-acting formulations should be performed prior to PRRT because they might interfere with receptor binding [4]. The treatment regimen of Lu-177-labeled PRRT for the noncompromised patient is that three to five cycles of 5.55-7.4 GBq $(150-200 \mathrm{mCi})$

Table 1 Physical properties of lutetium-177

\begin{tabular}{|c|c|c|c|c|c|c|c|c|}
\hline $\begin{array}{l}\text { Nuclide } \\
\text { symbol }\end{array}$ & $\begin{array}{l}\text { Atomic } \\
\text { number }\end{array}$ & Half-life & $\begin{array}{l}\text { Decay } \\
\text { modes }\end{array}$ & $\begin{array}{l}\text { Daughter } \\
\text { isotope }\end{array}$ & $\begin{array}{l}\mathrm{E}_{\beta-} \\
\max \mathrm{meV}\end{array}$ & $\mathrm{E} \gamma(\%) \mathrm{keV}$ & Production Method & $\begin{array}{l}\text { Tissue penetration } \text { max }_{(\mathrm{mm})} \\
(\mathrm{m})\end{array}$ \\
\hline Lu-177 & 71 & 6.7 days & $\beta^{-}$ & ${ }^{177} \mathrm{Hf}$ & 0.5 & $113(6.4), 208(11)$ & $\begin{array}{l}{ }_{71}^{176} \mathrm{Lu}(\mathrm{n}, \gamma){ }_{71}^{177} \mathrm{Lu},{ }_{70}^{176} \mathrm{Yb}(\mathrm{n}, \gamma){ }_{70}^{177} \mathrm{Yb} \\
\quad \rightarrow{ }_{71}^{177} \mathrm{Lu}\end{array}$ & 2 \\
\hline
\end{tabular}


activity with 6-12 weeks of time interval [4]. The current standard treatment protocols require confinement of patients to specialized shielded hospital radiation wards for 1-3 days after each administration of $7.4 \mathrm{GBq}(200 \mathrm{mCi}) \mathrm{Lu}-177$ labeled PRRT $[25,26]$. The outpatient protocol is also proposed for Lu-177-based PRRT for NETs [27, 28].

\section{Dosimetry}

Due to emission of $\gamma$-radiation of $208 \mathrm{keV}$ of Lu-177, it is possible to perform quantitative imaging and to estimate the absorbed dose calculation [24]. According to the recent studies, the whole-body dose was $0.03-0.07 \mathrm{mGy} / \mathrm{MBq}$ for $\mathrm{Lu}-$ 177-labeled PRRT [24, 29], the absorbed renal dose is 0.6$1.2 \mathrm{mGy} / \mathrm{MBq}$ and the dosimetric analysis of the spleen was 0.7-1.8 mGy/MBq [24, 29, 30]. Bone marrow absorbed a dose of radiation that ranged from 0.02 to $0.07 \mathrm{mGy} / \mathrm{MBq}$ $[24,31]$. Those dosimetric calculations of the absorbed bone marrow and kidney dose support the idea that more than 2 Gy seems appropriate for PRRT with Lu-177-labeled PRRT, because the dose-limiting organs are bone marrow and kidneys [31]. However, these results should be carefully applied to practical clinical settings because the published results were very different using the various methods for calculating absorbed dose for the tissue [24, 29-32]. Additionally, the activity quantifications using planar image were dependent on the background correction, careful placing of a background region of interest (ROI) is needed for the correct dosimetry [32]. The pretreatment dosimetry of Lu-177-labeled PRRT based on whole-body diagnostic scans could therefore be utilized to tailor therapy with more accuracy [32].

\section{Toxicity}

\section{Nephrotoxicity}

The pattern of nephrotoxicity after PRRNT is that of progressive chronic renal disease [30]. Glomerular cells are relatively radiosensitive and not able to regenerate; therefore all radiolabeled therapies bear a high nephrotoxic potential [19]. Renal irradiation is not SSTR mediated, but related primarily to the very rapid clearance of the small radiopeptides that are filtered through the glomeruli and reabsorbed by the tubular cells with subsequent radiation to the glomeruli, which leads to the nephrotoxicity $[19,33]$. It is reported that the grade 4 renal toxicity was occurred in $0.4 \%$ of patients after treatment with Lu-177-labeled PRRT $[16,34]$ and the PRRT leads to a reduction in kidney function with a loss of creatinine clearance of 4\% per year using Lu-177-based PRRT [35]. However, if adequate renal protection and fractionation described above are applied, nephrotoxicity could be mild [4]. According to a consensus protocol, the calculated maximally tolerated absorbed dose to the kidneys is $27 \mathrm{~Gy}$ when Lu-177-labeled PRRT is administered as three or four fractions at intervals of 8-10 weeks [36]. Renal dose evaluation for Lu-177-labeled PRRT is significantly lower when compared with Y-90labeled peptides, which means that $\mathrm{Lu}-177$ is potentially less nephrotoxic [30, 33, 37].

\section{Myelotoxicity}

Although acute or subacute bone marrow toxicity seems to be severe (grade 3 and 4), it is mostly reversible. In comparison with Y-90, it is assumed that the prevalence of acute myelotoxicity is less in patients with a treatment cycle utilizing Lu-177-labeled PRRT (2-3\% vs 10-13\%) [14, 31, 33, $38-41]$. Subacute hematological toxicities of grades 3 and 4 were observed in $2-11 \%$ of patients receiving $\mathrm{Lu}-177$-labeled PRRT [14, 31, 33, 38-41]. It is reported that the nadir normally occurs 4-6 weeks after each treatment, followed by a recovery phase [31]. Nevertheless, reduced bone marrow reserve, and more infrequently, sporadic cases of myelodysplastic syndrome or overt acute myelogenous leukemia have been reported [16, 33, 34, 42]. The patients with baseline nephrotoxicity (transient or persistent elevation in creatinine) tend to suffer from more hematological toxicity after PRRT [33]. Both prolonged circulation time of Lu-177labeled PRRT and a poor renal function are the most important factors that explain the increased toxicity to the bone marrow [43]. It is controversial whether the prior chemotherapy is a risk factor for hematologic toxicity or not $[14,31,33]$.

\section{Hormonal Crises}

A so-called carcinoid crisis is a syndrome related to the sudden massive release of the metabolically active hormones and receptor stimulation, which could occur spontaneously after manipulation of the respective functional tumors [44-46]. The symptoms include hypertension, hypotension, diarrhea, bronchoconstriction, arrhythmia and electrolyte imbalance and it is a medical emergency [46]. It is reported that the hormonal crises after Lu-177-based PRRT occur infrequently and all patients with a hormonal crisis eventually recovered [46].

\section{Others}

Other less serious side effects, such as nausea, vomiting, and abdominal pain, are more common and can be controlled by supportive care [46]. The infusion of amino acid for renal protection could cause nausea and vomiting [4]. 


\section{Treatment Response and Survival Analyses}

PRRT with the somatostatin analogues labeled with Lu-177 has been explored in NETs for more than a decade. Though the reported result of PRRT using Lu-177 is relatively small, it seems that the response to Lu-177-based PRRT appears better than Y-90-labeled counterpart [16, 34, 35, 42]. The first study of Lu-177 described the treatment response for 35 patients with gastroenteropancreatic NETs who had a follow-up at 3-6 months after receiving the final dose of Lu-177 DOTA-TATE therapy [11]. In the study by Kwekkeboom et al. [41], Lu-177-labeled PRRT showed a survival benefit of 40-72 months from diagnosis, compared with historical controls. The Lu-177 DOTA-TATE for progressive midgut NETs resulted in marked longer progression-free survival than other octreotide treatment in recent randomized phase 3 trial [47]. The published clinical studies have reported that the patients with NETs treated with Lu-177 DOTATATE have a radiological objective response rate (defined as complete remission and partial remission) of 9-35\% and the gastroenteropancreatic NETs show the best objective responses (with partial responses ranging from 9 to $29 \%$ and complete remission from 2 to $6 \%$ ) [26, 36, 48]. Survival analyses using PRRT have shown that patients with high SSTR expression and tumors with low $(<2 \%)$ or moderate $(2-20 \%)$ Ki67 proliferation index have significantly higher objective responses that translate into significantly longer survival [41, 42]. The objective tumor response rate with $\mathrm{Lu}-177$-labeled DOTA-TATE is summarized in Table 2.

\section{Future of Lu-177-labeled PRRT}

Additional application for Y-90-based PRRT have been reported for SSTR-positive tumors including meningiomas, medulloblastomas or astrocytomas and the results are regarded as encouraging for survival $[42,49]$. There are also several clinical trials to apply PRRT using Y-90 to medullary thyroid carcinoma and refractory dedifferentiated thyroid carcinomas; however, the reported results were less favorable [50-52]. Those reported studies evaluated treatment response in SSTR-positive tumors with Y-90-labeled PRRT not with Lu177. For more extended indications for Lu-177-labeled PRRT, various clinical trials are needed.

Combinations of with Lu-177-labeled peptides with Y-90labeled peptides or with chemotherapeutic agent are being actively investigated and may prove to be of additional therapeutic benefit. Initial data indicate that combination treatments with the two isotopes of Y-90 and Lu-177 linked either to DOTA-TOC or to DOTA-TATE administered in sequential treatment cycles or as a cocktail infusion for several cycles improve survival $[53,54]$. It seems that combination of Lu177-labeled somatostatin analogues and Y-90 labeled somatostatin analogues has the most effective treatment response in

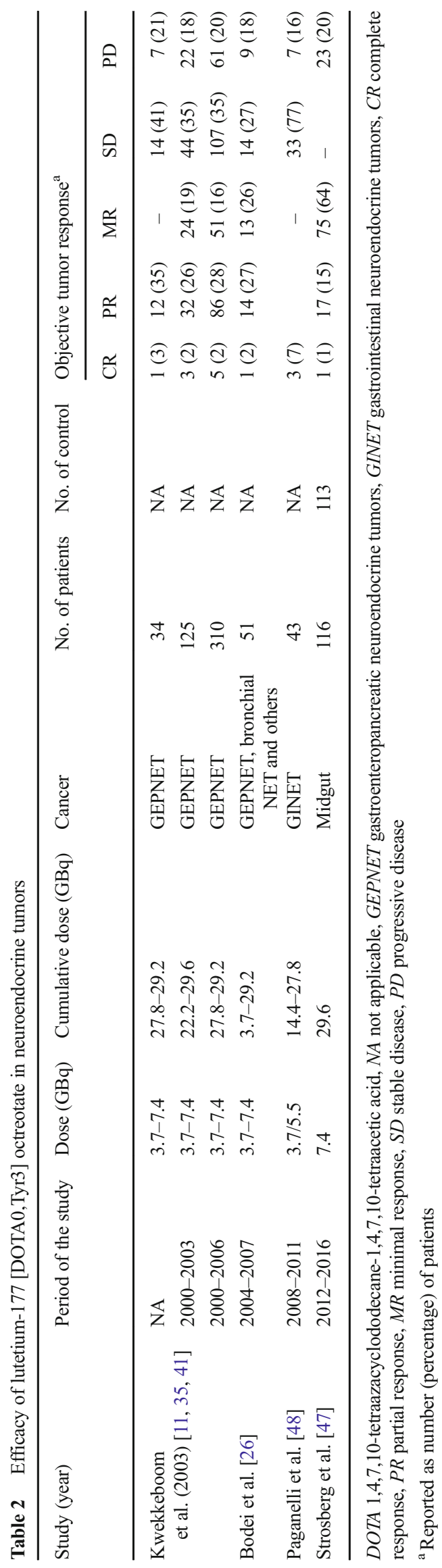


patients with variously sized or small metastases [55]. The clinical studies in patients with advanced low-grade NETs to assess the safety and efficacy of a combination of Lu-177 DOTA-TATE with chemotherapy including everolimus, capecitabine or temozolomide as peptide receptor chemoradionuclide therapies have been conducted [56-58]. The clinical utility of peptide receptor chemoradionuclide therapy for the patients with unresectable locally advanced NETs was investigated and it was revealed that it has a potential role as a neoadjuvant therapy [59]. Though there are some advantages of the combination therapies, efficacy of combination of Y-90- and Lu-177-labeled analogues or peptide receptor chemoradiotherapy should be evaluated via larger prospective randomized trials to confirm these results.

The scatchard analyses are in progress to develop new PRRTs labeled with Lu-177 which have higher affinity for the SSTR [60-62]. Additionally, there was a trial of intraoperative somatostatin receptor detection with Y-90 and Lu-177 DOTA-TOC using handled gamma probe and it is concluded that gamma probe-guided surgery after Lu-177 PRRT was feasible for more complete tumor resection [63].

\section{Conclusion}

In the management of rare neuroendocrine malignancies, radiolabeled peptides have made their greatest impact. Especially as 87-92\% of NETs express SSTR2, PRRT should be considered as a therapeutic option or a supportive care for the patients with advanced NETs [64]. Diagnostic and radiotherapeutic targeting of NETs with Lu-177-labeled peptide-based nuclear probes has been proved useful. Treatment with Lu-177-labeled somatostatin analogues is a more promising new tool compared with Y-90-loaded counterparts or other various treatments in the management of patients with inoperable or metastasized NETs. Several studies reported the promising results of the PRRT using Lu-177; however, the size of the studies is still limited. Therefore, large comparative studies are required for direct assessment of the distinct efficacies of Lu-177-labeled PRRT. To fully exploit its potential, the development of new radiopeptides and optimal combination regimen with improved pharmacokinetics is warranted. In addition to clinical research about combination therapies of analogues labeled with various radionuclides or chemoradionuclide therapy, efforts to upregulate somatostatin receptor expression or to develop the radiosensitizer are also required.

\section{Compliance with Ethical Standards}

Conflict of Interest Keunyoung Kim and Seong-Jang Kim have no conflicts of interest related to the current study.
Ethical Approval This article does not contain any studies with human participants performed by any of the authors.

Informed Consent This article does not contain any studies with human participants performed by any of the authors.

\section{References}

1. Fraenkel M, Kim MK, Faggiano A, Valk GD. Epidemiology of gastroenteropancreatic neuroendocrine tumours. Best Pract Res Clin Gastroenterol. 2012;26:691-703.

2. Yao JC, Hassan M, Phan A, Dagohoy C, Leary C, Mares JE, et al. One hundred years after "carcinoid": epidemiology of and prognostic factors for neuroendocrine tumors in 35,825 cases in the United States. J Clin Oncol. 2008;26:3063-72.

3. Moller LN, Stidsen CE, Hartmann B, Holst JJ. Somatostatin receptors. Biochim Biophys Acta. 1616;2003:1-84.

4. Bodei L, Mueller-Brand J, Baum RP, Pavel ME, Horsch D, O'Dorisio MS, et al. The joint IAEA, EANM, and SNMMI practical guidance on peptide receptor radionuclide therapy (PRRNT) in neuroendocrine tumours. Eur J Nucl Med Mol Imaging. 2013;40: 800-16.

5. Kulke MH, Shah MH, Benson AB III, Bergsland E, Berlin JD, Blaszkowsky LS, et al. Neuroendocrine tumors, version 1.2015. J Natl Compr Cancer Netw. 2015;13:78-108.

6. Modlin IM, Oberg K, Chung DC, Jensen RT, de Herder WW, Thakker RV, et al. Gastroenteropancreatic neuroendocrine tumours. Lancet Oncol. 2008;9:61-72.

7. Ramage JK, Davies AH, Ardill J, Bax N, Caplin M, Grossman A, et al. Guidelines for the management of gastroenteropancreatic neuroendocrine (including carcinoid) tumours. Gut. 2005;54(Suppl 4): iv1-16.

8. Metz DC, Jensen RT. Gastrointestinal neuroendocrine tumors: pancreatic endocrine tumors. Gastroenterology. 2008;135:1469-92.

9. Susini C, Buscail L. Rationale for the use of somatostatin analogs as antitumor agents. Ann Oncol. 2006;17:1733-42.

10. Janson ET, Oberg K. Long-term management of the carcinoid syndrome. Treatment with octreotide alone and in combination with alpha-interferon. Acta Oncol. 1993;32:225-9.

11. Kwekkeboom DJ, Bakker WH, Kam BL, Teunissen JJ, Kooij PP, de Herder WW, et al. Treatment of patients with gastro-enteropancreatic (GEP) tumours with the novel radiolabelled somatostatin analogue [177Lu-DOTA(0),Tyr3] octreotate. Eur J Nucl Med Mol Imaging. 2003;30:417-22.

12. Okarvi SM. Recent developments in 99Tcm-labelled peptide-based radiopharmaceuticals: an overview. Nucl Med Commun. 1999;20: 1093-112.

13. de Jong M, Breeman WA, Bernard BF, Bakker WH, Schaar M, van Gameren A, et al. [177Lu-DOTA(0),Tyr3] octreotate for somatostatin receptor-targeted radionuclide therapy. Int J Cancer. 2001;92: 628-33.

14. Delpassand ES, Samarghandi A, Zamanian S, Wolin EM, Hamiditabar M, Espenan GD, et al. Peptide receptor radionuclide therapy with $177 \mathrm{Lu}$-DOTATATE for patients with somatostatin receptor-expressing neuroendocrine tumors: the first US phase 2 experience. Pancreas. 2014;43:518-25.

15. Maecke HR, Reubi JC. Somatostatin receptors as targets for nuclear medicine imaging and radionuclide treatment. J Nucl Med. 2011;52:841-4.

16. Kwekkeboom DJ, Mueller-Brand J, Paganelli G, Anthony LB, Pauwels S, Kvols LK, et al. Overview of results of peptide receptor radionuclide therapy with 3 radiolabeled somatostatin analogs. J Nucl Med. 2005;46(Suppl 1):62s-6s. 
17. Cremonesi M, Botta F, Di Dia A, Ferrari M, Bodei L, De Cicco C, et al. Dosimetry for treatment with radiolabelled somatostatin analogues. A review. Q J Nucl Med Mol Imaging. 2010;54:37-51.

18. Kam BL, Teunissen JJ, Krenning EP, de Herder WW, Khan S, van Vliet EI, et al. Lutetium-labelled peptides for therapy of neuroendocrine tumours. Eur J Nucl Med Mol Imaging. 2012;39(Suppl 1): S103-12.

19. Kulkarni HR, Schuchardt C, Baum RP. Peptide receptor radionuclide therapy with (177)Lu labeled somatostatin analogs DOTATATE and DOTATOC: contrasting renal dosimetry in the same patient. Recent Results Cancer Res. 2013;194:551-9.

20. Bodei L, Cremonesi M, Ferrari M, Pacifici M, Grana CM, Bartolomei M, et al. Long-term evaluation of renal toxicity after peptide receptor radionuclide therapy with 90Y-DOTATOC and 177Lu-DOTATATE: the role of associated risk factors. Eur J Nucl Med Mol Imaging. 2008;35:1847-56.

21. Watkins J. Reactions to gelatin plasma expanders. Lancet. 1994;344:328-9. author reply 9-30

22. Rolleman EJ, Melis M, Valkema R, Boerman OC, Krenning EP, de Jong M. Kidney protection during peptide receptor radionuclide therapy with somatostatin analogues. Eur J Nucl Med Mol Imaging. 2010;37:1018-31.

23. Barron ME, Wilkes MM, Navickis RJ. A systematic review of the comparative safety of colloids. Arch Surg. 2004;139:552-63.

24. Wehrmann C, Senftleben S, Zachert C, Muller D, Baum RP. Results of individual patient dosimetry in peptide receptor radionuclide therapy with $177 \mathrm{Lu}$ DOTA-TATE and 177Lu DOTA-NOC. Cancer Biother Radiopharm. 2007;22:406-16.

25. Bakker WH, Breeman WA, Kwekkeboom DJ, De Jong LC, Krenning EP. Practical aspects of peptide receptor radionuclide therapy with [177Lu][DOTA0, Tyr3]octreotate. Q J Nucl Med Mol Imaging. 2006;50:265-71.

26. Bodei L, Cremonesi M, Grana CM, Fazio N, Iodice S, Baio SM, et al. Peptide receptor radionuclide therapy with (1)(7)(7)LuDOTATATE: the IEO phase I-II study. Eur J Nucl Med Mol Imaging. 2011;38:2125-35.

27. Calais PJ, Turner JH. Radiation safety of outpatient $177 \mathrm{Lu}-$ octreotate radiopeptide therapy of neuroendocrine tumors. Ann Nucl Med. 2014;28:531-9.

28. Olmstead C, Cruz K, Stodilka R, Zabel P, Wolfson R. Quantifying public radiation exposure related to lutetium-177 octreotate therapy for the development of a safe outpatient treatment protocol. Nucl Med Commun. 2015;36:129-34.

29. Schuchardt C, Kulkarni HR, Prasad V, Zachert C, Muller D, Baum RP. The Bad Berka dose protocol: comparative results of dosimetry in peptide receptor radionuclide therapy using (177) $\mathrm{Lu}-$ DOTATATE, (177)Lu-DOTANOC, and (177)Lu-DOTATOC. Recent Results Cancer Res. 2013;194:519-36.

30. Valkema R, Pauwels SA, Kvols LK, Kwekkeboom DJ, Jamar F, de Jong $\mathrm{M}$, et al. Long-term follow-up of renal function after peptide receptor radiation therapy with (90)Y-DOTA(0),Tyr(3)-octreotide and (177)Lu-DOTA(0), Tyr(3)-octreotate. J Nucl Med. 2005;46(Suppl 1):83s-91s.

31. Bergsma H, Konijnenberg MW, Kam BL, Teunissen JJ, Kooij PP, de Herder WW, et al. Subacute haematotoxicity after PRRT with (177)Lu-DOTA-octreotate: prognostic factors, incidence and course. Eur J Nucl Med Mol Imaging. 2016;43:453-63.

32. Garkavij M, Nickel M, Sjogreen-Gleisner K, Ljungberg M, Ohlsson T, Wingardh K, et al. 177Lu-[DOTA0,Tyr3] octreotate therapy in patients with disseminated neuroendocrine tumors: analysis of dosimetry with impact on future therapeutic strategy. Cancer. 2010;116:1084-92.

33. Bodei L, Kidd M, Paganelli G, Grana CM, Drozdov I, Cremonesi $\mathrm{M}$, et al. Long-term tolerability of PRRT in 807 patients with neuroendocrine tumours: the value and limitations of clinical factors. Eur J Nucl Med Mol Imaging. 2015;42:5-19.
34. Bodei L, Ferone D, Grana CM, Cremonesi M, Signore A, Dierckx RA, et al. Peptide receptor therapies in neuroendocrine tumors. J Endocrinol Investig. 2009;32:360-9.

35. Kwekkeboom DJ, Kam BL, van Essen M, Teunissen JJ, van Eijck $\mathrm{CH}$, Valkema $\mathrm{R}$, et al. Somatostatin-receptor-based imaging and therapy of gastroenteropancreatic neuroendocrine tumors. Endocr Relat Cancer. 2010;17:R53-73.

36. Kwekkeboom DJ, Teunissen JJ, Bakker WH, Kooij PP, de Herder WW, Feelders RA, et al. Radiolabeled somatostatin analog [177LuDOTA0,Tyr3]octreotate in patients with endocrine gastroenteropancreatic tumors. J Clin Oncol. 2005;23:2754-62.

37. Bodei L, Cremonesi M, Zoboli S, Grana C, Bartolomei M, Rocca P, et al. Receptor-mediated radionuclide therapy with 90Y-DOTATOC in association with amino acid infusion: a phase I study. Eur J Nucl Med Mol Imaging. 2003;30:207-16.

38. Pfeifer AK, Gregersen T, Gronbaek H, Hansen CP, Muller-Brand J, Herskind Bruun K, et al. Peptide receptor radionuclide therapy with Y-DOTATOC and (177)Lu-DOTATOC in advanced neuroendocrine tumors: results from a Danish cohort treated in Switzerland. Neuroendocrinology. 2011;93:189-96.

39. Sabet A, Ezziddin K, Pape UF, Ahmadzadehfar H, Mayer K, Poppel T, et al. Long-term hematotoxicity after peptide receptor radionuclide therapy with $177 \mathrm{Lu}$-octreotate. J Nucl Med. 2013;54:1857-61.

40. Gupta SK, Singla S, Bal C. Renal and hematological toxicity in patients of neuroendocrine tumors after peptide receptor radionuclide therapy with $177 \mathrm{Lu}$-DOTATATE. Cancer Biother Radiopharm. 2012;27:593-9.

41. Kwekkeboom DJ, de Herder WW, Kam BL, van Eijck CH, van Essen M, Kooij PP, et al. Treatment with the radiolabeled somatostatin analog [177 Lu-DOTA 0,Tyr3]octreotate: toxicity, efficacy, and survival. J Clin Oncol. 2008;26:2124-30.

42. Imhof A, Brunner P, Marincek N, Briel M, Schindler C, Rasch H, et al. Response, survival, and long-term toxicity after therapy with the radiolabeled somatostatin analogue [90Y-DOTA]-TOC in metastasized neuroendocrine cancers. J Clin Oncol. 2011;29:2416-23.

43. Svensson J, Berg G, Wangberg B, Larsson M, Forssell-Aronsson E, Bernhardt P. Renal function affects absorbed dose to the kidneys and haematological toxicity during (1)(7)(7)Lu-DOTATATE treatment. Eur J Nucl Med Mol Imaging. 2015;42:947-55.

44. Karmy-Jones R, Vallieres E. Carcinoid crisis after biopsy of a bronchial carcinoid. Ann Thorac Surg. 1993;56:1403-5.

45. Kharrat HA, Taubin H. Carcinoid crisis induced by external manipulation of liver metastasis. J Clin Gastroenterol. 2003;36:87-8.

46. de Keizer B, van Aken MO, Feelders RA, de Herder WW, Kam BL, van Essen M, et al. Hormonal crises following receptor radionuclide therapy with the radiolabeled somatostatin analogue $[177 \mathrm{Lu}-$ DOTA0,Tyr3] octreotate. Eur J Nucl Med Mol Imaging. 2008;35: 749-55.

47. Strosberg J, El-Haddad G, Wolin E, Hendifar A, Yao J, Chasen B, et al. Phase 3 trial of $177 \mathrm{Lu}$-Dotatate for Midgut Neuroendocrine Tumors. N Engl J Med. 2017;376:125-35.

48. Paganelli G, Sansovini M, Ambrosetti A, Severi S, Monti M, Scarpi E, et al. $177 \mathrm{Lu}$-Dota-octreotate radionuclide therapy of advanced gastrointestinal neuroendocrine tumors: results from a phase II study. Eur J Nucl Med Mol Imaging. 2014;41:1845-51.

49. Bartolomei M, Bodei L, De Cicco C, Grana CM, Cremonesi M, Botteri E, et al. Peptide receptor radionuclide therapy with (90)YDOTATOC in recurrent meningioma. Eur J Nucl Med Mol Imaging. 2009;36:1407-16.

50. Bodei L, Handkiewicz-Junak D, Grana C, Mazzetta C, Rocca P, Bartolomei $\mathrm{M}$, et al. Receptor radionuclide therapy with $90 \mathrm{Y}-$ DOTATOC in patients with medullary thyroid carcinomas. Cancer Biother Radiopharm. 2004;19:65-71.

51. Iten F, Muller B, Schindler C, Rasch H, Rochlitz C, Oertli D, et al. [(90)Yttrium-DOTA]-TOC response is associated with survival 
benefit in iodine-refractory thyroid cancer: long-term results of a phase 2 clinical trial. Cancer. 2009;115:2052-62.

52. Iten F, Muller B, Schindler C, Rochlitz C, Oertli D, Macke HR, et al. Response to [90Yttrium-DOTA]-TOC treatment is associated with long-term survival benefit in metastasized medullary thyroid cancer: a phase II clinical trial. Clin Cancer Res. 2007;13:6696702.

53. Villard L, Romer A, Marincek N, Brunner P, Koller MT, Schindler $\mathrm{C}$, et al. Cohort study of somatostatin-based radiopeptide therapy with [(90)Y-DOTA]-TOC versus [(90)Y-DOTA]-TOC plus [(177)Lu-DOTA]-TOC in neuroendocrine cancers. J Clin Oncol. 2012;30:1100-6.

54. Kunikowska J, Krolicki L, Hubalewska-Dydejczyk A, Mikolajczak R, Sowa-Staszczak A, Pawlak D. Clinical results of radionuclide therapy of neuroendocrine tumours with 90Y-DOTATATE and tandem 90Y/177Lu-DOTATATE: which is a better therapy option? Eur J Nucl Med Mol Imaging. 2011;38:1788-97.

55. De Jong M, Valkema R, Jamar F, Kvols LK, Kwekkeboom DJ, Breeman WA, et al. Somatostatin receptor-targeted radionuclide therapy of tumors: preclinical and clinical findings. Semin Nucl Med. 2002;32:133-40.

56. Claringbold PG, Price RA, Turner JH. Phase I-II study of radiopeptide $177 \mathrm{Lu}$-octreotate in combination with capecitabine and temozolomide in advanced low-grade neuroendocrine tumors. Cancer Biother Radiopharm. 2012;27:561-9.

57. Claringbold PG, Turner JH. NeuroEndocrine tumor therapy with Lutetium-177-octreotate and Everolimus (NETTLE): a phase I study. Cancer Biother Radiopharm. 2015;30:261-9.
58. Kamp K, Gumz B, Feelders RA, Kwekkeboom DJ, Kaltsas G, Costa FP, et al. Safety and efficacy of everolimus in gastrointestinal and pancreatic neuroendocrine tumors after (177)Lu-octreotate. Endocr Relat Cancer. 2013;20:825-31.

59. Barber TW, Hofman MS, Thomson BN, Hicks RJ. The potential for induction peptide receptor chemoradionuclide therapy to render inoperable pancreatic and duodenal neuroendocrine tumours resectable. Eur J Surg Oncol. 2012;38:64-71.

60. Cescato R, Waser B, Fani M, Reubi JC. Evaluation of $177 \mathrm{Lu}-$ DOTA-sst2 antagonist versus 177Lu-DOTA-sst2 agonist binding in human cancers in vitro. J Nucl Med. 2011;52:1886-90.

61. Fani M, Braun F, Waser B, Beetschen K, Cescato R, Erchegyi J, et al. Unexpected sensitivity of sst2 antagonists to N-terminal radiometal modifications. J Nucl Med. 2012;53:1481-9.

62. Wild D, Fani M, Fischer R, Del Pozzo L, Kaul F, Krebs S, et al. Comparison of somatostatin receptor agonist and antagonist for peptide receptor radionuclide therapy: a pilot study. J Nucl Med. 2014;55:1248-52.

63. Todorovic-Tirnanic M, Kaemmerer D, Prasad V, Hommann M, Baum RP. Intraoperative somatostatin receptor detection after peptide receptor radionuclide therapy with $(177) \mathrm{Lu}-$ and $(90) \mathrm{Y}$ DOTATOC (tandem PRRNT) in a patient with a metastatic neuroendocrine tumor. Recent Results Cancer Res. 2013;194:487-96.

64. Reubi JC. Peptide receptors as molecular targets for cancer diagnosis and therapy. Endocr Rev. 2003;24:389-427. 